\title{
Effect of HHH-Therapy on Regional CBF after Severe Subarachnoid Hemorrhage Studied by Bedside Xenon-Enhanced CT
}

\author{
Henrik Engquist ${ }^{1,2}$ (D) Elham Rostami ${ }^{1} \cdot$ Elisabeth Ronne-Engström $^{1} \cdot$ \\ Pelle Nilsson $^{1} \cdot$ Anders Lewén $^{1} \cdot$ Per Enblad $^{1}$
}

Published online: 5 October 2017

(C) The Author(s) 2017. This article is an open access publication

\begin{abstract}
Background Management of delayed cerebral ischemia (DCI) following subarachnoid hemorrhage (SAH) is difficult and still carries controversies. In this study, the effect of therapeutic hypervolemia, hemodilution, and hypertension (HHH-therapy) on cerebral blood flow (CBF) was assessed by xenon-enhanced computerized tomography (XeCT) hypothesizing an increase in $\mathrm{CBF}$ in poorly perfused regions.

Methods Bedside XeCT measurements of regional CBF in mechanically ventilated SAH patients were routinely scheduled for day $0-3,4-7$, and 8-12. At clinical suspicion of DCI, patients received 5-day HHH-therapy. For inclusion, XeCT was required at $0-48 \mathrm{~h}$ before start of $\mathrm{HHH}$ (baseline) and during therapy. Data from corresponding time-windows were also collected for non-DCI patients.

Results Twenty patients who later developed DCI were included, and twenty-eight patients without DCI were identified for comparison. During $\mathrm{HHH}$, there was a slight nonsignificant increase in systolic blood pressure (SBP) and a significant reduction in hematocrit. Median global cortical CBF for the DCI group increased from 29.5 (IQR 24.6-33.9) to 38.4 (IQR 27.0-41.2) $\mathrm{ml} / 100 \mathrm{~g} / \mathrm{min}$ $(P=0.001)$. There was a concomitant increase in regional $\mathrm{CBF}$ of the worst vascular territories, and the proportion of
\end{abstract}

Henrik Engquist

henrik.engquist@akademiska.se

1 Dept of Neuroscience/Neurosurgery, Uppsala University, Uppsala, Sweden

2 Dept of Surgical Sciences/Anesthesia and Intensive Care, Uppsala University Hospital, 75185 Uppsala, Sweden area with blood flow below $20 \mathrm{ml} / 100 \mathrm{~g} / \mathrm{min}$ was significantly reduced. Non-DCI patients showed higher CBF at baseline, and no significant change over time.

Conclusions HHH-therapy appeared to increase global and regional CBF in DCI patients. The increase in SBP was small, while the decrease in hematocrit was more pronounced, which may suggest that intravascular volume status and rheological effects are of importance. XeCT may be potentially helpful in managing poor-grade $\mathrm{SAH}$ patients.

Keywords Subarachnoid hemorrhage (SAH) .

Delayed cerebral ischemia (DCI) .

Cerebral blood flow (CBF) · HHH-therapy (Triple-H) .

Xenon CT $(\mathrm{XeCT})$

\section{Introduction}

The complex mechanisms leading to secondary brain injury and the sometimes devastating course of delayed cerebral ischemia (DCI) [1] after aneurysmal subarachnoid hemorrhage (SAH) are still not well understood [2]. Improved techniques for early surgical or endovascular interventions and general improvement of the neurointensive care (NIC) have reduced mortality and morbidity in these patients during the last decades [3]. Still there is little evidence supporting prophylactic strategies to minimize the risk of DCI and also concerning therapeutic interventions when DCI is suspected [4]. In modern NIC, clinical surveillance combined with multimodal systemic and cerebral monitoring is used to aid the early detection of avoidable factors and also DCI [5]. There is, however, no consensus regarding the best tools to guide the therapy at suspicion of DCI [6]. 
After early repair of the aneurysm, most guidelines propose fluid therapy to ensure normovolemia or mild hypervolemia, monitoring of blood pressure and vasoactive support if necessary to avoid hypotension [7, 8]. At suspicion of DCI, therapeutic hypervolemia, hemodilution, and hypertension (HHH-therapy) [9] has been widely used aiming to increase cerebral blood flow (CBF) in ischemic regions. Controversy exists concerning the actual effects of the different components of this intervention [10]. In the standard NIC protocol for SAH patients at Uppsala University Hospital, therapeutic interventions on suspicion of DCI include cautious institution of HHH-therapy with moderate blood pressure targets and frequent reevaluation to avoid side effects and complications.

The aim of this study was to evaluate the effects of HHH-therapy on CBF, testing the hypothesis that HHHtherapy increases global $\mathrm{CBF}$ and also regional $\mathrm{CBF}$ $(\mathrm{rCBF})$ in areas at risk of ischemia. Bedside xenon-enhanced computerized tomography (XeCT) was used to assess $\mathrm{rCBF}$ prior to clinical suspicion of DCI and during the treatment to resolve DCI.

\section{Materials and Methods}

\section{Patients}

Patients diagnosed with severe spontaneous SAH admitted to Uppsala University Hospital during the time period 2013-2016 were prospectively enrolled in the study. The diagnosis of SAH was verified by CT. As ventilator treatment is a prerequisite for bedside $\mathrm{XeCT}$ in our setting, only patients requiring intubation due to their neurological state at admission or neurological deterioration day 0-1 were included, i.e., patients with more severe SAH. Exclusion criteria were severe intracranial hypertension, deep sedation with thiopental, respiratory problems requiring $\mathrm{FiO}_{2}$ $>0.6$, the absence of informed consent, and futility or "do not resuscitate" order.

\section{CBF Measurements: protocol}

According to our standard NIC protocol, CBF measurements were scheduled for three time intervals: day $0-3$, $4-7$, and 8-12. Patients with clinical diagnose of DCI received HHH-therapy as described below. For inclusion in this study, a XeCT measurement within $0-48 \mathrm{~h}$ before the start of HHH-therapy was required (used as baseline), and the intention was to perform the next XeCT measurement during ongoing therapy when logistically possible. Data were also collected for non-DCI patients with corresponding XeCT measurements in the same time-windows as a reference group for comparison.

\section{Xenon CT CBF Measurements: Method}

The XeCT procedures in our NIC unit are conducted as described by Gur et al. and Yonas et al. [11-14], using inhaled xenon as an inert contrast agent during the CT scans. Since xenon is radiopaque, lipid soluble, and crosses the blood brain barrier, its concentration in the cerebral tissue can be measured by CT. Blood flow calculation is based on Kety's $[15,16]$ application of the Fick principle; blood flow is proportional to inert gas uptake in tissue. Mobile equipment allows bedside measurements in the NIC unit, which facilitates the clinical use of XeCT [17]. Stable (non-radioactive) xenon at a concentration of $28 \%$ is administered to the patients breathing circuit for $4.5 \mathrm{~min}$ using the Enhancer 3000 and computer software (Diversified Diagnostic Products Inc, Huston, USA). CT scans during the xenon inhalation are obtained by the CereTom (Neurologica, Boston, USA). The xenon delivery and CT scans are synchronized by the computer software, and the resulting radiologic tissue enhancement of the xenon washin enables CBF to be calculated $(\mathrm{ml} / 100 \mathrm{~g} / \mathrm{min})$ and plotted as color-coded maps in scans at four different levels of the brain (eight scans per level, two at baseline and six enhanced during xenon wash-in). Levels with extensive artifacts from bone structures or coils/clips were excluded from calculation. Typically, three scan levels could be used for further calculations in each patient. Mean blood flow in each of twenty evenly distributed cortical regions of interest (ROIs) was calculated (Fig. 1) for every level [13], resulting in a total of sixty ROIs. Typical area of each ROI was $350-450 \mathrm{~mm}^{2}$. ROIs in areas of hematoma or with artifacts from ventricular drains were excluded.

\section{Neurointensive Care}

Management of all patients followed our standard NIC procedures for SAH as earlier described by Ryttlefors et al. [5], where the cornerstones are continuous multimodal monitoring of physiological and biochemical parameters and cautious clinical observation for avoidable factors with prompt interventions to minimize secondary brain injury. Sedation with propofol $\left(\right.$ Propolipid $^{\circledR}$, Fresenius Kabi AB, Uppsala, Sweden) is titrated in the interval $0-4 \mathrm{mg} / \mathrm{kg} / \mathrm{h}$, and morphine (Morfin, Meda AB, Solna, Sweden) is administered as needed. Patients with suspicion of hydrocephalus or altered level of consciousness receive a ventriculostomy catheter for monitoring of intracranial pressure (ICP) and drainage of cerebrospinal fluid. Elevated ICP $(>20 \mathrm{mmHg})$ is treated with open ventricular drainage against a pressure level of $15 \mathrm{mmHg}$. The general policy is to treat aneurysms early with endovascular coil embolization as first choice when possible or surgical clipping. After the aneurysm repair, patients are kept 

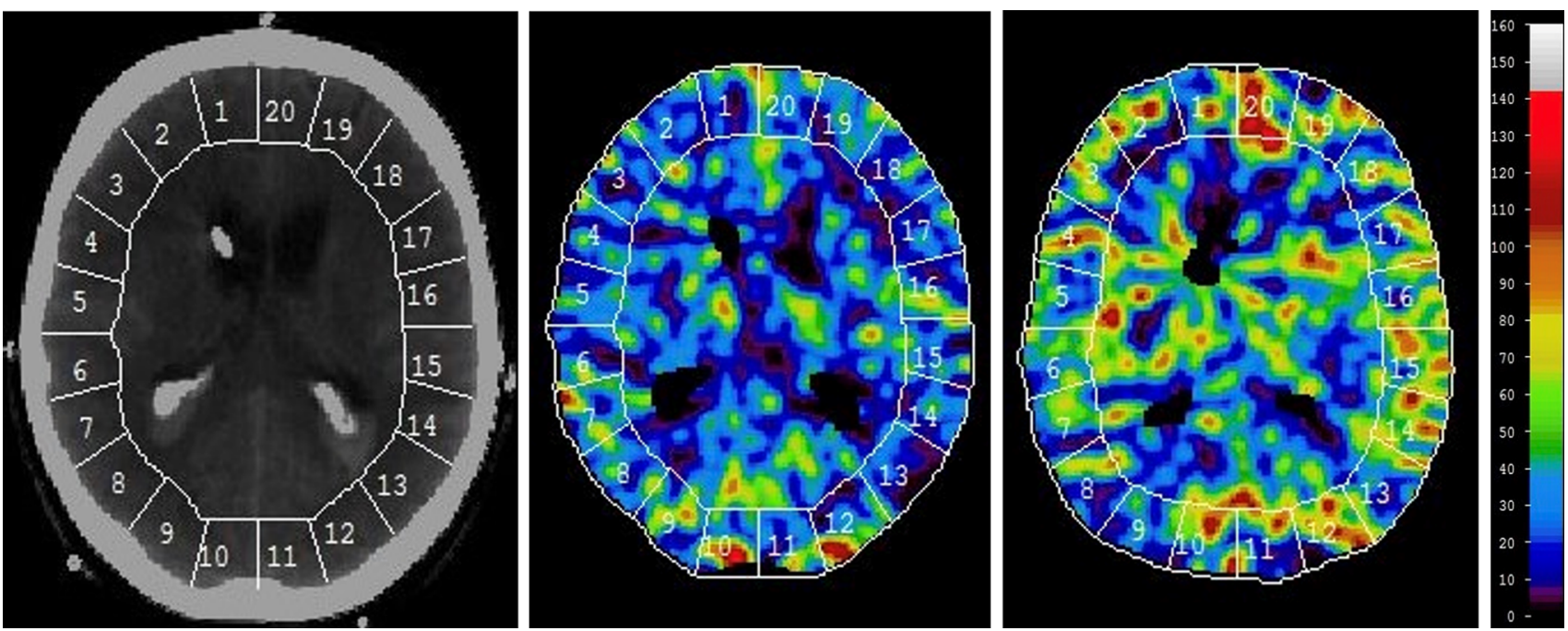

Fig. 1 Example of XeCT measurements before and during $\mathrm{HHH}-$ therapy (one of three scan levels). Global and regional cortical CBF $(\mathrm{ml} / 100 \mathrm{~g} / \mathrm{min})$ was calculated as mean of CBF in the corresponding regions of interest (ROIs) at three scan levels of the brain for each patient. Global $\mathrm{CBF}-\mathrm{ROI}$ 1-20. Right anterior cerebral artery

mildly hypervolemic if not contraindicated by intracranial mass effect or elevated ICP. The volume status is maintained by fluid administration in the higher normal range with addition of albumin infusion (Flexbumin ${ }^{\circledR} 200 \mathrm{mg} / \mathrm{ml}$, Baxter AG, Vienna, Austria) if needed and monitored by central venous pressure, clinical evaluation and rigorous fluid balance calculation. Nimodipine $\left(\right.$ Nimotop $^{\circledR}$, Bayer Pharma AG, Berlin, Germany) is given from day one and onwards.

\section{Detection of DCI and HHH-Therapy}

To detect DCI, patients were monitored for general and focal neurological changes by repeated wake-up tests [18]. In the case of neurological deterioration, $\mathrm{CT}$ and laboratory tests were performed to rule out intracranial mass, manifest infarction, hydrocephalus, and meningitis. If DCI was concluded in the absence of other causes of deterioration, HHH-therapy to augment CBF was initiated for 5 days if not contraindicated by the intracranial situation, congestive heart failure, fluid overload or severe respiratory problems. Patients received daily infusions of $500 \mathrm{ml}$ dextran solution (Rheomacrodex ${ }^{\circledR}$, Meda AB, Solna, Sweden) and $200 \mathrm{ml}$ albumin (Flexbumin ${ }^{\circledR} 200 \mathrm{mg} / \mathrm{ml}$, Baxter AG, Vienna, Austria) and were kept in supine position. In our standard NIC protocol, emphasis is on avoiding severe side effects and the blood pressure targets during HHH-therapy are moderate. The systolic blood pressure (SBP) was closely monitored and kept above $140 \mathrm{mmHg}$ using vasoactive agents if needed. As first line, dobutamine (Hospira, Lake
territory-ROI 1-2, right middle cerebral artery territory-ROI 3-8, right posterior cerebral artery territory-ROI 9-10 and equally for ROI 11-20 on the left side. $\mathrm{CBF}$ cerebral blood flow, $\mathrm{HHH}$ therapy therapeutic hypervolemia, hemodilution and hypertension, $\mathrm{XeCT}$ xenon-enhanced computerized tomography

Forest, IL, USA) was used for inotropy or, if insufficient, norepinephrine (Noradrenalin, Hospira Nordic AB, Stockholm, Sweden) was added as vasopressor. The clinical effect of the HHH-therapy was repeatedly evaluated.

\section{XeCT Parameters}

Global cortical CBF (ml/100 g/min) was calculated as mean of all ROIs at all scan levels used (Fig. 1). Regional CBF for each vascular territory was calculated as mean of the corresponding ROIs at all scan levels used (Fig. 1): right anterior cerebral artery-ROI 1-2, right middle cerebral artery-ROI 3-8, right posterior cerebral arteryROI 9-10 and equally for ROI $11-20$ on the left side. The vascular territory with lowest CBF was identified as the worst vascular territory in each patient.

To detect and quantify the extent of areas with low blood flow and near-ischemic flow, thresholds for local CBF were set to 20 and $10 \mathrm{ml} / 100 \mathrm{~g} / \mathrm{min}[19,20]$. The percentage of cortical ROI area with local CBF below these thresholds was then calculated as sum of ROI area $\left(\mathrm{mm}^{2}\right)$ with local CBF below the threshold divided by the total analyzed ROI area in each patient.

\section{Short-Term Outcome Parameters}

Clinical course outcome was defined as neurological state at discharge from the NIC unit and determined as good (responding to commands, GCS motor 6), poor (unconscious, GCS motor $\leq 5$ ), or dead. 
Cerebral infarcts visible on follow-up CT at 12 days or later after admission to the NIC unit were categorized as $<20 \mathrm{~mm}, 20-39 \mathrm{~mm},>40 \mathrm{~mm}$, or multiple.

\section{Statistical Methods}

SPSS statistics 23.0 software (IBM Corp, Armonk, NY, USA) was used for statistical analyses of the collected data. Differences in systemic physiological parameters between measurements were tested by paired samples $t$ test. CBF
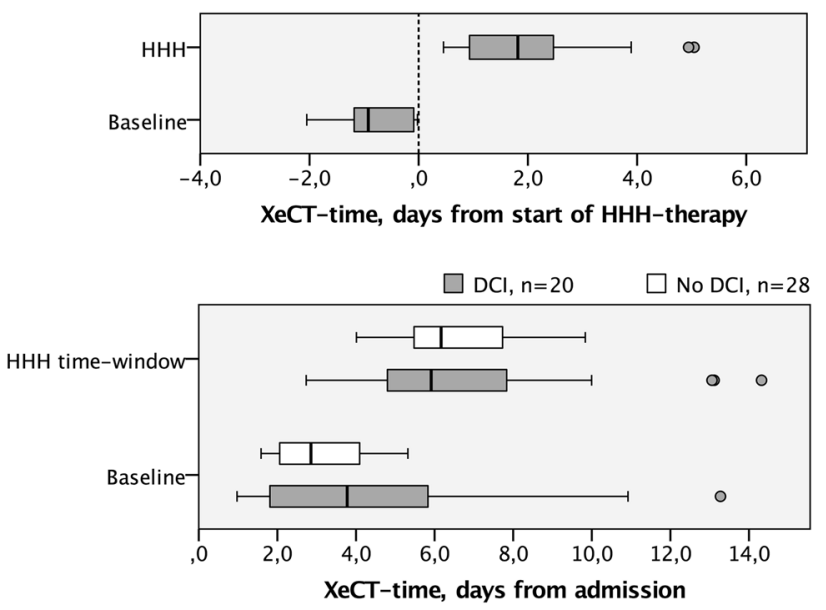

Fig. 2 Time for XeCT measurements in relation to the start of HHHtherapy for DCI patients (above). Time for XeCT at baseline and during HHH-therapy in relation to admission to neurosurgical intensive care for DCI patients (gray) and for non-DCI patients (white) at corresponding time-windows (below). DCI delayed cerebral ischemia, HHH-therapy therapeutic hypervolemia, hemodilution and hypertension, $\mathrm{XeCT}$ xenon-enhanced computerized tomography data for groups of patients are presented as median values and interquartile range because of non-normal distribution. Differences in CBF parameters between related samples were tested by Wilcoxon signed-ranks test, and between independent samples by Mann-Whitney $U$ test. Statistical significance level was set at $P<0.05$.

\section{Results}

A total of 119 intubated SAH patients underwent one or more XeCT measurements during the time period 2013-2016. During this period, 20 patients with clinical suspicion of DCI were identified where XeCT measurements of $\mathrm{CBF}$ had been taken within $0-48 \mathrm{~h}$ before the initiation of HHH-therapy (baseline), and during the 5-day HHH-therapy (Fig. 2). Twenty-eight SAH patients with no suspicion of DCI, who had corresponding XeCT measurements in the same timewindows, were identified as a group for comparison of the natural course (Fig. 2). The characteristics of these groups are presented in Table 1. The median time-point for initiation of HHH-therapy was 4.38 days (IQR 2.75-5.97) after the admission to the NIC unit.

\section{Hemodynamics and Ventilation}

Hemodynamic parameters and ventilation were clinically stable during the $\mathrm{XeCT}$ procedures. The alterations from start to end in all procedures $(N=96)$ were small; mean arterial pressure (MAP) mean $92.5 \mathrm{mmHg}$ (CI 90.0-95.0) versus 89.9 (CI 87.6-92.2), ICP mean $14.3 \mathrm{mmHg}$ (CI 13.2-15.4) versus 14.4 (CI 13.5-15.4), $\mathrm{PCO}_{2}$ mean
Table 1 Characteristics of patients in the DCI group who received $\mathrm{HHH}$-therapy and the non-DCI group

\begin{tabular}{|c|c|c|c|c|c|}
\hline \multirow{2}{*}{\multicolumn{2}{|c|}{ Characteristic }} & \multirow{3}{*}{$\begin{array}{l}\begin{array}{l}\text { DCI } \\
n\end{array} \\
20\end{array}$} & \multirow{3}{*}{$(\%)$} & \multirow{3}{*}{$\begin{array}{l}\begin{array}{l}\text { No DCI } \\
\mathrm{n}\end{array} \\
28\end{array}$} & \multirow{3}{*}{$(\%)$} \\
\hline & & & & & \\
\hline Patients & $\mathrm{n}$ & & & & \\
\hline Gender & $\mathrm{F}$ & 12 & $(60)$ & 19 & (68) \\
\hline Age, years & Mean [range] & 58 & {$[40-75]$} & 60 & [28-84] \\
\hline \multirow[t]{5}{*}{ Hunt and Hess grade at admission/first $\mathrm{XeCT}^{1}$} & I & $0 / 0$ & & $2 / 0$ & $(7 / 0)$ \\
\hline & II & $5 / 0$ & $(25 / 0)$ & $1 / 0$ & $(4 / 0)$ \\
\hline & III & $5 / 1$ & $(25 / 5)$ & $7 / 8$ & $(25 / 29)$ \\
\hline & IV & $7 / 18$ & $(35 / 90)$ & $14 / 18$ & $(50 / 64)$ \\
\hline & $\mathrm{V}$ & $3 / 1$ & $(15 / 5)$ & $4 / 2$ & $(14 / 7)$ \\
\hline \multirow[t]{3}{*}{ CT Fisher group } & 2 & 0 & & 1 & (4) \\
\hline & 3 & 6 & (30) & 5 & (18) \\
\hline & 4 & 14 & (70) & 22 & (79) \\
\hline \multirow[t]{3}{*}{ Treatment modality } & Endovascular & 18 & (90) & 18 & (64) \\
\hline & Surgical clip & 1 & (5) & 9 & (32) \\
\hline & None & 1 & (5) & 1 & (4) \\
\hline
\end{tabular}

${ }^{1}$ Neurological state according to Hunt and Hess assessed both at admission and at the time of the first XeCT. DCI delayed cerebral ischemia, HHH-therapy therapeutic hypervolemia, hemodilution and hypertension, $\mathrm{XeCT}$ xenon-enhanced computerized tomography 
$5.41 \mathrm{kPa}$ (CI 5.29-5.53) versus 5.51 (CI 5.40-5.63). No adverse events were observed in relation to the procedures.

SBP, MAP, cerebral perfusion pressure (CPP), $\mathrm{pCO}_{2}$, and hematocrit at start of the $\mathrm{XeCT}$ measurements at the different time-windows for the DCI and non-DCI groups are presented in Table 2. The mean SBP was slightly elevated during HHH-therapy in the DCI group, from $151.2 \mathrm{mmHg}$ (CI $142.1-160.3$ ) to $157.3 \mathrm{mmHg}$ (CI 150.7-163.8), but the difference did not reach statistical significance. Among the DCI patients, hematocrit was significantly reduced during HHH-therapy compared to baseline (Table 2).

The use of inotropes and vasopressors was low in all groups. The number of patients and dose range for each group are also presented in Table 2.

\section{Global Cortical CBF}

Results of the XeCT measurements are presented in Table 2 and Fig. 3. Global cortical CBF at baseline was significantly lower in the DCI group compared to the nonDCI group; median $29.5 \mathrm{ml} / 100 \mathrm{~g} / \mathrm{min}$ (IQR 24.6-33.9) versus 34.9 (IQR 29.0-41.7) $(P=0.005)$.

During HHH-therapy, the DCI group showed an increase in median global cortical CBF from 29.5 (IQR 24.6-33.9) to 38.4 (IQR 27.0-41.2) $\mathrm{ml} / 100 \mathrm{~g} / \mathrm{min}$ $(P=0.001)$, while no significant change over time was seen in the non-DCI group. No statistical analyses were performed for differences between DCI patients during HHH-therapy and non-DCI patients at the corresponding time-window.

Table 2 Systemic hemodynamic parameters, ventilation, sedation, and vasoactive medication at the time of XeCT measurements (upper part). Calculated XeCT CBF parameters (lower part)

\begin{tabular}{|c|c|c|c|c|c|c|c|c|c|c|c|}
\hline & \multicolumn{3}{|c|}{ DCI $(n=20)$ Baseline } & \multicolumn{3}{|c|}{ DCI During $\mathrm{HHH}$} & \multicolumn{3}{|c|}{ No DCI $(n=28)$ Baseline } & \multicolumn{2}{|c|}{ No DCI Day 5-8 } \\
\hline & Mean & \multicolumn{2}{|l|}{ (CI) } & Mean & \multicolumn{2}{|l|}{ (CI) } & \multicolumn{2}{|c|}{ Mean } & $(\mathrm{CI})$ & Mean & (CI) \\
\hline SBP mmHg & 151.2 & \multirow{2}{*}{\multicolumn{2}{|c|}{$\begin{array}{l}(142.1-160.3) \\
P=0.105\end{array}$}} & \multirow[t]{2}{*}{157.3} & \multirow{2}{*}{\multicolumn{2}{|c|}{$(150.7-163.8)$}} & \multirow{2}{*}{\multicolumn{2}{|c|}{150.0}} & \multirow[t]{2}{*}{$(143.5-156.4)$} & 152.9 & $(145.9-159.8)$ \\
\hline & & & & & & & & & & & \\
\hline MAP mmHg & 95.4 & \multicolumn{2}{|c|}{$(87.1-103.7)$} & 94.0 & \multicolumn{2}{|c|}{$(88.7-99.4)$} & \multicolumn{2}{|c|}{90.3} & $(87.0-93.6)$ & 92.5 & $(88.2-96.7)$ \\
\hline $\mathrm{CPP} \mathrm{mmHg}$ & 80.9 & \multicolumn{2}{|c|}{$(72.4-89.3)$} & 79.7 & \multicolumn{2}{|c|}{$(74.3-85.1)$} & \multicolumn{2}{|c|}{78.6} & $(74.3-82.9)$ & 79.0 & $(74.8-83.3)$ \\
\hline Hematocrit $\%$ & 36.4 & \multirow{2}{*}{\multicolumn{2}{|c|}{$\begin{array}{l}(34.7-38.0) \\
P<0.001 *\end{array}$}} & \multirow[t]{2}{*}{31.7} & \multirow{2}{*}{\multicolumn{2}{|c|}{$(30.2-33.2)$}} & \multirow{2}{*}{\multicolumn{2}{|c|}{33.9}} & \multirow[t]{2}{*}{$(32.6-35.3)$} & 32.5 & $(31.4-33.6)$ \\
\hline & & & & & & & & & & & \\
\hline $\mathrm{pCO}_{2} \mathrm{kPa}$ & 5.20 & \multicolumn{2}{|c|}{$(4.96-5.44)$} & 5.34 & $(5.10$ & $5.57)$ & 5.35 & & $(5.14-5.57)$ & 5.71 & $(5.45-5.97)$ \\
\hline Sedation dose, propofol $\mathrm{mg} / \mathrm{kg} / \mathrm{h}$ & 2.58 & $(2.15-$ & $3.02)$ & 2.57 & $(2.16-$ & 2.98) & 2.70 & & $(2.13-3.26)$ & 2.81 & $(2.22-3.40)$ \\
\hline & & & [range] & $\mathrm{n}$ & {$[\mathrm{ra}$} & ge] & & $\mathrm{n}$ & [range] & $\mathrm{n}$ & [range] \\
\hline Dobutamine, $\mathrm{n}$ [range ug/kg/min] & & & $-]$ & 5 & & $-12.0]$ & & 4 & {$[1.6-4.0]$} & 3 & {$[1.0-6.2]$} \\
\hline Norepinephrine, $\mathrm{n}$ [range ug/kg/ & & & {$[0.06]$} & 2 & & $5-0.15]$ & & 3 & {$[0.01-0.12]$} & 2 & {$[0.05-0.08]$} \\
\hline & & Median & (IQR) & & Median & (IQR) & & Median & (IQR) & Median & (IQR) \\
\hline glob CBF ml/100 g/min & & 29.5 & $(24.6-$ & 33.9) & 38.4 & $(27.0-41$. & $.2)$ & 34.9 & $(29.0-41.7)$ & 36.5 & $(28.0-42.3)$ \\
\hline & & & $P=0$ & $001 *$ & & & & & & & \\
\hline$\% \mathrm{ROI}$ area $[\mathrm{rCBF}<20 \mathrm{ml} / 100$ & $\mathrm{g} / \mathrm{min}]$ & 26.2 & $(13.4-$ & 14.5) & 8.55 & $(2.4-34.8$ & & 11.9 & $(3.2-23.0)$ & 9.1 & $(2.1-29.2)$ \\
\hline & & & $P=0$ & $019 *$ & & & & & & & \\
\hline$\% \mathrm{ROI}$ area $[\mathrm{rCBF}<10 \mathrm{ml} / 100$ & $\mathrm{g} / \mathrm{min}]$ & 6.7 & $(0.0-1$ & l.0) & 0.0 & $(0.0-5.0)$ & & 0.7 & $(0.0-4.6)$ & 0.7 & $(0.0-4.9)$ \\
\hline & & & $P=0$ & 056 & & & & & & & \\
\hline rCBF worst territory $\mathrm{ml} / 100 \mathrm{~g} / \mathrm{min}$ & & 19.6 & $(15.0-$ & 24.2) & 27.3 & $(17.8-34$ & $.1)$ & 27.2 & $(20.8-35.4)$ & 25.8 & $(17.4-31.4)$ \\
\hline & & & $P=0$ & $006^{*}$ & & & & & & & \\
\hline Index [rCBFworst/best hemisph] & & 0.59 & $(0.46-$ & $.75)$ & 0.70 & $(0.55-0.8$ & 83) & 0.70 & $(0.60-0.79)$ & 0.63 & $(0.52-0.73)$ \\
\hline & & & $P=0$ & $040 *$ & & & & & $P=0.029^{*}$ & & \\
\hline
\end{tabular}

* Indicates $P<0.05$. $C B F$ cerebral blood flow, $C I$ confidence interval, $C P P$ cerebral perfusion pressure, $D C I$ delayed cerebral ischemia, $H H H$ therapy therapeutic hypervolemia, hemodilution and hypertension, $I Q R$ interquartile range, $M A P$ mean arterial pressure, $r C B F$ regional cerebral blood flow, $R O I$ region of interest, $S B P$ systolic blood pressure 
Fig. 3 Boxplots of global cortical $\mathrm{CBF}$ and regional $\mathrm{CBF}$ of the worst vascular territory for patients clinically diagnosed with DCI at baseline and during $\mathrm{HHH}$-therapy, and patients with no suspicion of DCI at corresponding time-windows (above). Proportion (\%) of ROI area with local $\mathrm{CBF}$ below thresholds of 20 and $10 \mathrm{ml} /$ $100 \mathrm{~g} / \mathrm{min}$, respectively, for DCI and non-DCI patients (below). CBF cerebral blood flow, $D C I$ delayed cerebral ischemia, $\mathrm{HHH}$ -

therapy therapeutic

hypervolemia, hemodilution and hypertension, ROI area region of interest area
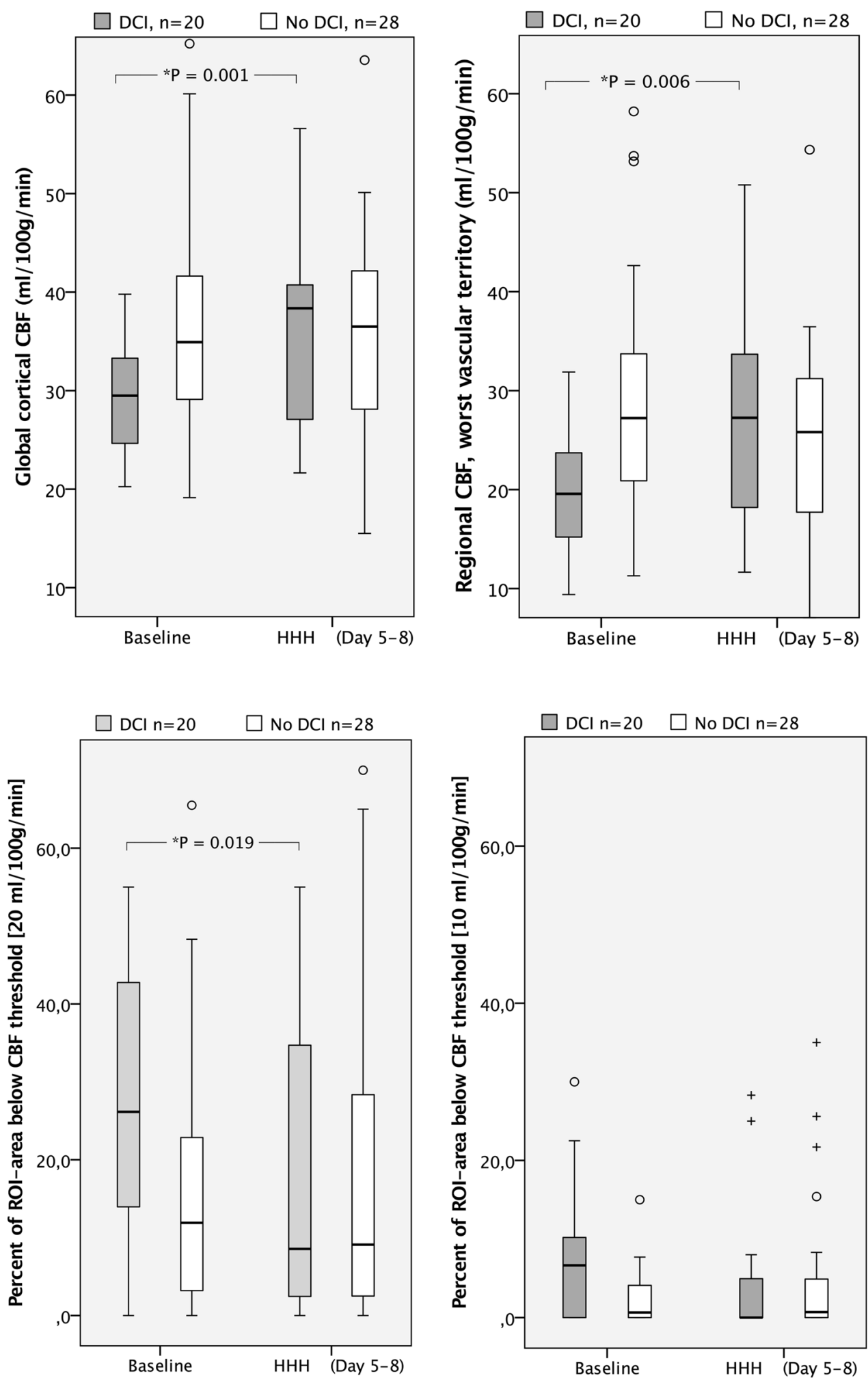

\section{Regional CBF and CBF Heterogeneity}

At baseline, there were also significant differences in regional CBF parameters between the DCI and non-DCI groups (Table 2; Fig. 3); median $\mathrm{rCBF}$ of the worst vascular territory for the DCI group was 19.6 (IQR 15.0-24.2) compared to $27.2(20.8-35.4) \mathrm{ml} / 100 \mathrm{~g} / \mathrm{min}$ for the non-DCI group $(P=0.005)$. Concerning regions with low or near-ischemic CBF, the DCI group had a larger proportion of ROI area with blood flow below $20 \mathrm{ml} /$ 
$100 \mathrm{~g} / \mathrm{min}$ (median 26.2 vs $11.9 \%, P=0.005$ ) and flow below $10 \mathrm{ml} / 100 \mathrm{~g} / \mathrm{min}$ (median 6.7 vs $0.7 \%, P=0.023$ ) compared to the non-DCI group.

During HHH-therapy, median $\mathrm{rCBF}$ of the worst vascular territory for the DCI group increased from 19.6 (IQR $15.0-24.2)$ to 27.3 (IQR 17.8-34.1) $\mathrm{ml} / 100 \mathrm{~g} / \mathrm{min}$ ( $P=0.006)$ (Table 2; Fig. 3), and the proportion of ROI area with local blood flow below the threshold of $20 \mathrm{ml} /$ $100 \mathrm{~g} / \mathrm{min}$ was reduced from median $26.2 \%$ at baseline to $8.55 \%(P=0.019)$. The area with flow below $10 \mathrm{ml} / 100 \mathrm{~g} /$ min was small already at baseline but tended to decrease (from 6.7 to $0.0 \%, P=0.056$ ). The relative blood flow in the worst vascular territory compared to the best hemispheric blood flow (CBF index of worst vascular territory) showed a small but statistically significant increase during HHH-therapy. In the non-DCI group, there were no significant changes from baseline to the second time-window regarding $\mathrm{rCBF}$ in the worst vascular territory or the proportion of ROI area with local blood flow below the specified thresholds. In this group, there was a small, but statistically significant reduction in the CBF index of worst vascular territory between the two measurements.

\section{Short-Term Outcome; Clinical Course and Cerebral Infarcts}

Short-term outcome parameters are presented in Fig. 4. The proportion of patients with favorable clinical course outcome was $65 \%$ in the HHH-treated DCI group and $57 \%$ in the non-DCI group. The proportion of patients where no infarcts $>20 \mathrm{~mm}$ were detected at follow-up CT was $65 \%$ in the DCI group and $46 \%$ in the non-DCI group. Since the non-DCI group is not considered a valid control group for HHH-therapy, no statistical analyses were performed on the differences in outcome parameters.

\section{Discussion}

Poor-grade, unconscious SAH patients still suffer high risk of deterioration and development of DCI during the acute course after the initial bleed, despite early aneurysm repair and modern specialized NIC. The main therapeutic option remains the use of one or several components of HHH-therapy. The effectiveness of the HHH-therapy has been questioned, and according to recent literature, the limited scientific support favors only augmentation of systemic blood pressure [10]. Moreover, the use of excessive fluid overload and high doses of vasopressors in these patients carries the risk of cardiopulmonary and cerebral complications [21-23].

The use of HHH-therapy to resolve DCI, according to our standardized NIC protocol, is instituted cautiously to avoid negative side effects, and the treatment is frequently reevaluated in each patient. Systemic blood pressure targets are moderate to avoid excessive use of vasopressors. The establishment of bedside $\mathrm{CBF} \mathrm{XeCT}$ in routine $\mathrm{NIC}$
Fig. 4 Clinical course outcome for patients in the DCI and nonDCI groups; neurological state at discharge from the NIC unit (good-responding to commands and GCS motor 6, poor-unconscious and GCS motor $\leq 5$, dead). Infarcts visible on follow-up CT $>$ day 12. $C T$ computerized tomography, $D C I$ delayed cerebral ischemia, GCS motor motor component of Glasgow coma scale, NIC neuro intensive care
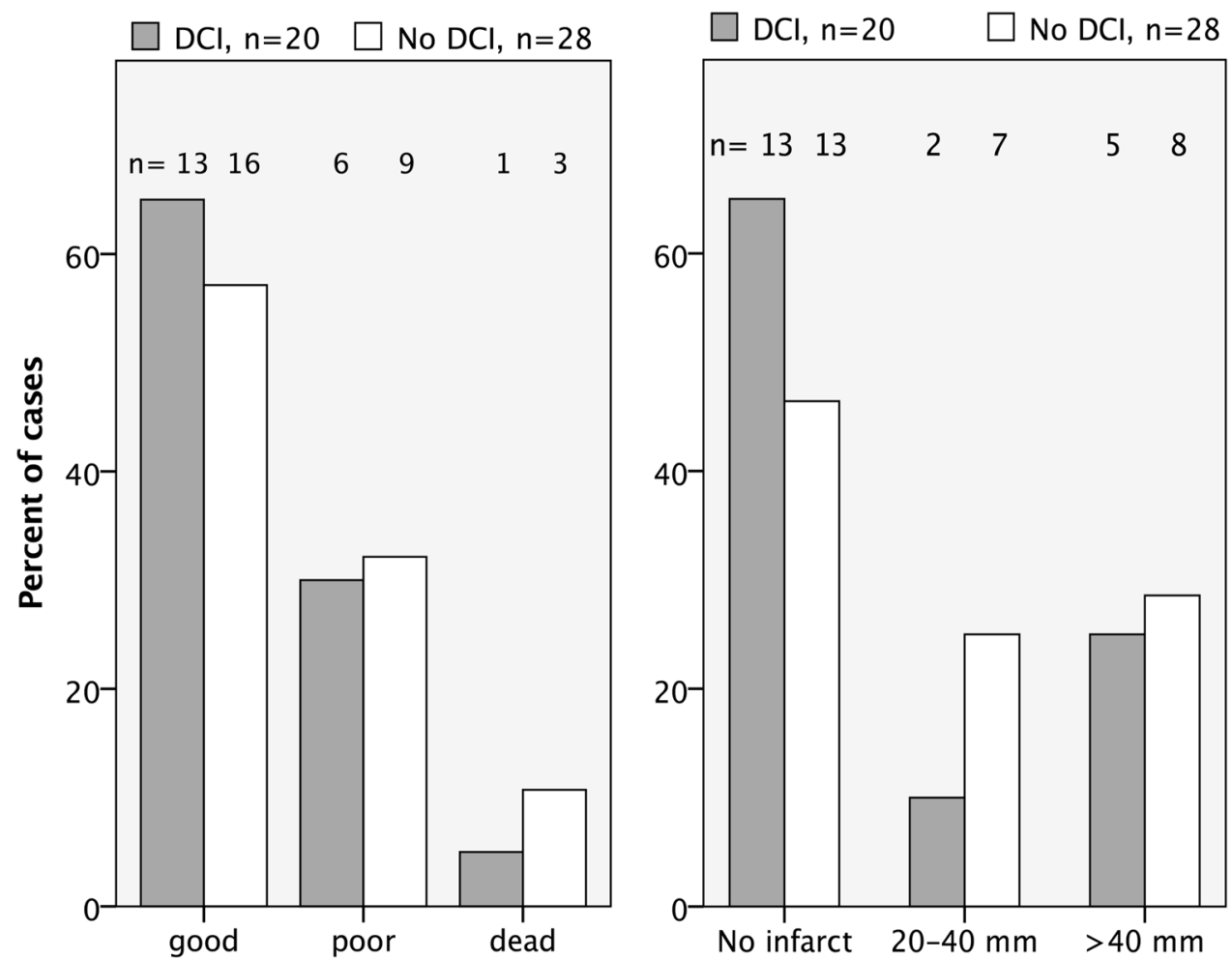
provided an excellent possibility to evaluate the effects of HHH-therapy on CBF in our setting. A general problem in the scientific evaluation of HHH-therapy is the difficulty to obtain a relevant control group, since it is unethical to leave one group of patients with signs of DCI without treatment. To overcome this shortcoming to some extent, we identified non-DCI patients with XeCT measurements in the same time-windows to serve as a reference group, although CBF dynamics in these patients may differ from patients with suspicion of DCI.

At baseline, the subsequently HHH-treated DCI patients had lower CBF than non-DCI patients. During HHH treatment there was a significant elevation of global CBF, whereas $\mathrm{CBF}$ in non-DCI patients remained unchanged over time. There is a possibility that the observed increase during $\mathrm{HHH}$-therapy may represent a natural spontaneous recovery of the initially low CBF, but there are several indications in this study that there was a real effect of the HHH-therapy on $\mathrm{CBF}$, which is discussed in the following sections.

The most important therapeutic goal is to achieve better perfusion in near-ischemic regions, and therefore, analysis of regional blood flow is of special importance. There was an increase in regional CBF in the previously worst perfused vascular regions during HHH-therapy, and the proportion of ROI area with blood flow below the threshold of $20 \mathrm{ml} / 100 \mathrm{~g} / \mathrm{min}$ decreased significantly. These findings indicate that the increase in $\mathrm{CBF}$ is not only luxury perfusion of already well-perfused regions, but also a true restoration of $\mathrm{CBF}$ in poorly perfused regions associated with HHH-therapy.

The restoration of CBF found in the group of patients receiving HHH-therapy does not necessarily correlate with better outcome. The absence of a strict DCI control group and the small number of patients make conclusions regarding outcome uncertain, and no long-term outcome data were analyzed. However, concerning short-term outcome, the frequencies of infarctions, and good clinical course outcome at discharge from the NIC unit were at similar levels for HHH-treated DCI patients and non-DCI patients. This observation also indicates that the $\mathrm{HHH}-$ therapy may have been effective.

One of the strengths of using XeCT with a mobile CTscanner is the possibility to conduct CBF measurements bedside, which minimizes alterations in the physiological situation of the patients. The hemodynamic and respiratory parameters proved to be stable in this study, and the amount of sedation given was low (Table 2), which indicates that the results obtained were not confounded by those factors.

From this study, it is difficult to assess which component of the HHH-therapy was most important for the observed increase in CBF. However, the results indicate that the controlled hypervolemia and resulting reduction in hematocrit may be of importance, since the elevation of SBP was small and CPP not significantly increased. This indication is in contrast with previous studies suggesting that the hypertensive treatment is most important [10], which calls for further studies.

In evaluating the effect of HHH-therapy, baseline measurement of CBF should ideally be performed immediately prior to the initiation of the therapy. However, this was not possible for logistic reasons and the risk of delaying treatment. Baseline CBF in our study thus represents measurements performed at $0-48 \mathrm{~h}$ prior to the start of HHH-therapy, which is not optimal but reasonably close to the start of therapy. The second CBF measurement, during the five-day therapy, was performed at median 1.82 days (range 0.45-5.04 days) after the start of HHHtherapy, suggesting a sustained increase in CBF after initiation of the therapy. Early and repeated measurements would be preferred to better evaluate a direct relationship between HHH-therapy and CBF changes, but an increased number of measurements might be questionable due to radiation exposure and also logistically difficult.

We find bedside $\mathrm{XeCT}$ feasible for clinical use in NIC to estimate $\mathrm{rCBF}$ in unconscious, mechanically ventilated patients, but the procedure takes the resources of a trained team of intensive care and radiology personnel. Regarding safety issues, XeCT using $28 \%$ inhaled xenon is previously reported safe [24], and all patients in our study were physiologically stable during the procedures. Since inert and rapidly washed-out xenon is used as contrast agent, the method allows repeated measurements, although taking radiation exposure into consideration.

\section{Conclusions}

The initial low global CBF found in patients diagnosed with DCI was significantly elevated during $\mathrm{HHH}$-therapy. A concomitant increase in $\mathrm{rCBF}$ was also found in the worst perfused regions and the proportion of ROI area with blood flow below the threshold of $20 \mathrm{ml} / 100 \mathrm{~g} / \mathrm{min}$ decreased significantly, which indicate a true effect of HHH-therapy. The increase in SBP was small, while the decrease in hematocrit was more pronounced, which may suggest that intravascular volume status and rheological effects are of importance. We found bedside XeCT to be a feasible and potentially clinically valuable tool in NIC.

\section{Compliance with Ethical Standards}

Conflict of interest The authors declare that they have no conflict of interest.

Ethical approval and informed consent The study protocol was approved by the Uppsala University Regional Ethical Review Board, 
and informed consent was obtained from the patients included or their next of kin. All procedures performed in the study were in compliance with the 1964 Helsinki Declaration and its later amendments or comparable ethical standards. The study was also approved by the local Radiation Safety Authority.

Open Access This article is distributed under the terms of the Creative Commons Attribution 4.0 International License (http:// creativecommons.org/licenses/by/4.0/), which permits unrestricted use, distribution, and reproduction in any medium, provided you give appropriate credit to the original author(s) and the source, provide a link to the Creative Commons license, and indicate if changes were made.

\section{References}

1. Frontera JA, Fernandez A, Schmidt JM, Claassen J, Wartenberg KE, Badjatia N, Connolly ES, Mayer SA. Defining vasospasm after subarachnoid hemorrhage: what is the most clinically relevant definition? Stroke. 2009;40(6):1963-8. doi:10.1161/ STROKEAHA.108.544700.

2. Carr KR, Zuckerman SL, Mocco J. Inflammation, cerebral vasospasm, and evolving theories of delayed cerebral ischemia. Neurol Res Int. 2013;2013:506584. doi:10.1155/2013/506584.

3. Ronne-Engström E, Enblad P, Gál G, Norbäck O, Ryttlefors M, Cesarini KG, Bolander H, Tovi M, Persson L. Patients with spontaneous subarachnoid haemorrhage-presentation of a 10-year hospital series. Br J Neurosurg. 2009;23(5):499-506. doi:10.1080/02688690902874901.

4. Brathwaite S, Macdonald RL. Current management of delayed cerebral ischemia: update from results of recent clinical trials. Transl Stroke Res. 2014;5(2):207-26. doi:10.1007/s12975-0130316-8.

5. Ryttlefors M, Howells T, Nilsson P, Ronne-Engström E, Enblad P. Secondary insults in subarachnoid hemorrhage: occurrence and impact on outcome and clinical deterioration. Neurosurgery. 2007;61(4):704-14. doi:10.1227/01.NEU.0000298898.38979.E3 discussion 714-705.

6. Sarrafzadeh AS, Vajkoczy P, Bijlenga P, Schaller K. Monitoring in neurointensive Care- the challenge to detect delayed cerebral ischemia in high-grade aneurysmal SAH. Front Neurol. 2014;5:134. doi:10.3389/fneur.2014.00134.

7. Connolly ES, Rabinstein AA, Carhuapoma JR, Derdeyn CP, Dion J, Higashida RT, Hoh BL, Kirkness CJ, Naidech AM, Ogilvy CS, Patel AB, Thompson BG, Vespa P, AHASCouncil, CoCRaIntervention, CoCNursing, CoCSaAnesthesia, CoCCardiology. Guidelines for the management of aneurysmal subarachnoid hemorrhage: a guideline for healthcare professionals from the American Heart Association/american Stroke Association. Stroke. 2012;43(6):1711-37. doi:10.1161/STR.0b013e3182587839.

8. Steiner T, Juvela S, Unterberg A, Jung C, Forsting M, Rinkel G, Organization ES. European stroke organization guidelines for the management of intracranial aneurysms and subarachnoid haemorrhage. Cerebrovasc Dis. 2013;35(2):93-112. doi:10.1159/ 000346087 .

9. Origitano TC, Wascher TM, Reichman OH, Anderson DE. Sustained increased cerebral blood flow with prophylactic hypertensive hypervolemic hemodilution ("triple-H" therapy) after subarachnoid hemorrhage. Neurosurgery. 1990;27(5):72939 discussion $\mathbf{7 3 9 - 7 4 0}$
10. Dankbaar JW, Slooter AJ, Rinkel GJ, Schaaf IC. Effect of different components of triple-H therapy on cerebral perfusion in patients with aneurysmal subarachnoid haemorrhage: a systematic review. Crit Care. 2010;14(1):R23. doi:10.1186/cc8886.

11. Gur D, Good WF, Wolfson SK Jr, Yonas H, Shabason L. In vivo mapping of local cerebral blood flow by xenon-enhanced computed tomography. Science (New York, NY). 1982;215(4537):1267-8.

12. Yonas H, Gur D, Wolfson SK, Good WF, Good BC, Latchaw RE. Xenon-enhanced computerised tomographic cerebral blood flow mapping. Lancet. 1984;1(8390):1357.

13. Yonas H, Darby JM, Marks EC, Durham SR, Maxwell C. CBF measured by Xe-CT: approach to analysis and normal values. J Cereb Blood Flow Metab. 1991;11(5):716-25. doi:10.1038/ jcbfm.1991.128.

14. Yonas H, Pindzola RP, Johnson DW. Xenon/computed tomography cerebral blood flow and its use in clinical management. Neurosurg Clin N Am. 1996;7(4):605-16.

15. Kety S, Schmidt C. The determination of cerebral blood flow in man by the use of nitrous oxide in low concentrations. Am J Physiol. 1945;143:53-66.

16. Kety SS. The measurement of cerebral blood flow by means of inert diffusible tracers. Keio J Med. 1994;43(1):9-14.

17. Sturnegk $\mathrm{P}$, Mellergård $\mathrm{P}$, Yonas $\mathrm{H}$, Theodorsson A, Hillman J. Potential use of quantitative bedside CBF monitoring (Xe-CT) for decision making in neurosurgical intensive care. Br J Neurosurg. 2007;21(4):332-9. doi:10.1080/02688690701411574.

18. Skoglund K, Hillered L, Purins K, Tsitsopoulos PP, Flygt J, Engquist H, Lewén A, Enblad P, Marklund N. The neurological wake-up test does not alter cerebral energy metabolism and oxygenation in patients with severe traumatic brain injury. Neurocrit Care. 2014;20(3):413-26. doi:10.1007/s12028-013-9876-4.

19. Fainardi E, Tagliaferri MF, Compagnone C, Tanfani A, Cocciolo F, Battaglia R, Frattarelli M, Pascarella R, Targa L, Chieregato A. Regional cerebral blood flow levels as measured by xenon-CT in vascular territorial low-density areas after subarachnoid hemorrhage are not always ischemic. Neuroradiology. 2006;48(9):685-90. doi:10.1007/s00234-006-0111-2.

20. Chieregato A, Tanfani A, Noto A, Fronza S, Cocciolo F, Fainardi E. Cerebral blood flow thresholds predicting new hypoattenuation areas due to macrovascular ischemia during the acute phase of severe and complicated aneurysmal subarachnoid hemorrhage: a preliminary study. Acta Neurochir Suppl. 2008;102:311-6.

21. Walid MS, Sahiner G, Robinson DR, Robinson JS. The relationship between pulmonary dysfunction and age in vasospasm patients receiving triple $\mathrm{H}$ therapy. $\mathrm{J}$ Vasc Interv Neurol. 2011;4(2):29-33.

22. Muhammad S, Güresir Á, Greschus S, Scorzin J, Vatter H, Güresir E. Posterior reversible encephalopathy syndrome as an overlooked complication of induced hypertension for cerebral vasospasm: systematic review and illustrative case. Stroke. 2016;47(2):519-22. doi:10.1161/STROKEAHA.115.011697.

23. Lee KH, Lukovits T, Friedman JA. "Triple-H" therapy for cerebral vasospasm following subarachnoid hemorrhage. Neurocrit Care. 2006;4(1):68-76. doi:10.1385/NCC:4:1:068.

24. Carlson AP, Brown AM, Zager E, Uchino K, Marks MP, Robertson C, Sinson GP, Marmarou A, Yonas H. Xenon-enhanced cerebral blood flow at $28 \%$ xenon provides uniquely safe access to quantitative, clinically useful cerebral blood flow information: a multicenter study. AJNR Am J Neuroradiol. 2011;32(7):1315-20. doi:10.3174/ajnr.A2522. 